\title{
Out-of-Plane Knife-Gate Microvalves for Controlling Large Gas Flows
}

\author{
Sjoerd Haasl, Member, IEEE, Stefan Braun, Anthony S. Ridgeway, Samir Sadoon, \\ Wouter van der Wijngaart, Member, IEEE, and Göran Stemme, Fellow, IEEE
}

\begin{abstract}
This paper considers design issues for microvalves for large gas flow control. It introduces out-of-plane knife-gate microvalves as a novel design concept and a proportional microvalve concept for pressure control applications. The design of three different actuator-gate configurations and first prototypes are presented. The first valve prototypes feature thermal silicon-aluminum bimorph actuators and the pressure-flow performance per chip area of the demonstrator valve presented is greatly increased using out-of-plane actuation and an out-of-plane orifice. The characterization of the actuators and of the pressure-flow performance is presented. The prototype valve allows for a flow change of $\Delta Q=3.4$ standard liters per minute (SLPM) at a pressure change of $\Delta P=95 \mathrm{kPa}\left(P_{\text {in }}=196.3 \mathrm{kPa}, P_{\text {out }}=101.3\right.$ kPa) on an active chip area of only $2.3 \times 3.7 \mathrm{~mm}^{2}$

[1515].
\end{abstract}

Index Terms-Microfluidics, micropneumatics, microvalves.

\section{INTRODUCTION}

$\mathbf{C}$ ONTROLLING a gas flow with microvalves provides a number of advantages. The main functional ones come from the rapid response time and low-power consumption offered by microsystems. Furthermore, microelectromechanical systems (MEMS) technology promises a cost-efficient approach through batch fabrication. The greatest advantage of microvalves, however, lies in the integration of the actuation mechanism with the other microvalve components, which enhances miniaturization and packaging. A representative overview of the vast amount of work established in this field can be found in [1].

However, gas microvalves are not used yet as standard components in industry because primarily of the cost-per-performance ratio of today's microvalves. The minimum actuator size is roughly determined by the force times stroke product required. Most microvalves are of the seat-valve type (diaphragm-type) illustrated in Fig. 1( $\alpha$ ), which requires a valve actuation that counteracts the large pressure forces controlled by the valve. Moreover, to allow for a large gas flow, a valve stroke of typically several tens of micrometers is needed. Microvalves manufactured with batch microfabrication techniques use either

This work was supported by Vinnova through the Summit framework. Subject Editor G. B. Hocker.

S. Haasl was with the Microsystem Laboratory, School of Electrical Engineering, Royal Institute of Technology (KTH), Stockholm SE-100 44, Sweden. $\mathrm{He}$ is now with IMEGO AB, Gothenburg SE-411 33, Sweden.

S. Braun, A. S. Ridgeway. S. Sadoon, W. van der Wijngaart, and G. Stemme are with the Microsystem Laboratory, School of Electrical Engineering, Royal Institute of Technology (KTH), Stockholm SE-100 44, Sweden (e-mail: stefan. braun@ee.kth.se).

Digital Object Identifier 10.1109/JMEMS.2006.880279 electrostatic actuation [2], pneumatic actuation [3], thermopneumatic actuation [4]-[6], or thermal (bimorph) actuation [7]. The actuator size, and thus the consumed valve footprint area for these devices, must be large in comparison to the minimum flow cross-sectional area, which prohibits large component counts per batch. Microvalves with actuation principles that require manual assembly include electrostatic actuation [8], [9], electromagnetic actuation [10], shape-memory alloy actuation [11], thermopneumatic actuation [12], and piezoelectric actuation [13], [14]. These valves offer larger energy density and larger stroke than do other techniques, however, cost more to assemble. Past attempts to minimize the required actuator force for membrane-type microvalves involve pressure balancing schemes [3], [15] or nozzle/seat optimization [16], [17].

Walters et al. [18] introduced gate microvalves, i.e., gas microvalves where an in-plane slider regulates an out-of-plane flow. The general principle is shown in Fig. $1(\beta)$. In this design, the static pressure and valve actuation are perpendicular ("cross-flow") and, therefore, do not counteract each other. However, gate-microvalve design comes at a cost. The limited actuation energy available in microsystems does not allow friction between sliding structures. Spacing between the flow orifice and the movable flow obstruction is, therefore, required. This means that leak flow occurs in the closed valve state. Fortunately, many valve applications, such as the pressure regulator design described below, tolerate leak flow. Gate-valve design removes a major actuator requirement and forms the basis for the novel knife-gate microvalves introduced in this paper.

\section{Design Issues}

The main issues involved in the design of a silicon micromachined valve are chip size and packaging. The chip size greatly affects the final cost, so the minimization of the footprint of the device is very important. When designing the nozzle and the actuator, one has to consider not only the required force and stroke length but also the size. These factors counteract each other, so a tradeoff is required. Another major factor in reducing the footprint area is the design of the pneumatic connections. Very often these are placed in the plane of the chip, so space is required, not only for the tubing itself but also for the fixation (gluing, welding) of the tubing to the chip. The packaging of the device is an extremely important and complicated issue and accounts for a large part of the production cost for most MEMS devices. For pneumatic MEMS devices, a key issue is leak tightness. This puts high demands on the materials used and limits the choices 


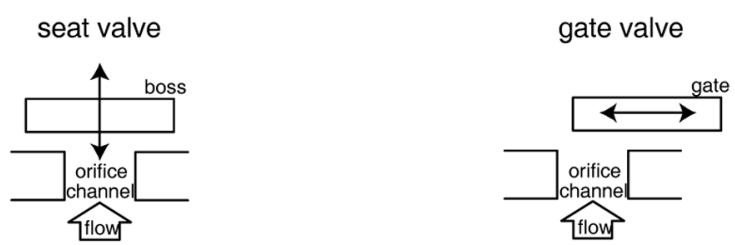

$(\alpha)$

( $\beta)$

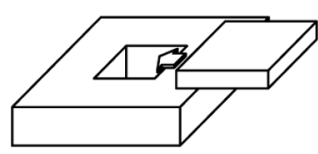

(a)

in plane gate orientation in plane gate movement out of plane flow literature $[18,19]$

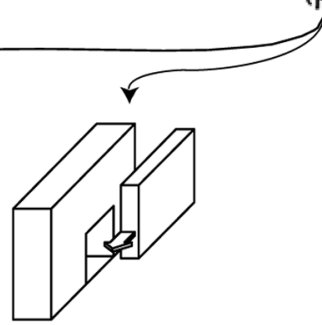

(b)

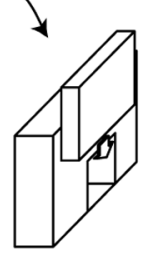

(c)

out of plane gate orientation out of plane gate orientation in plane gate movement out of plane gate movement in plane flow literature $[21,22]$ in plane flow literature [this work, 20]

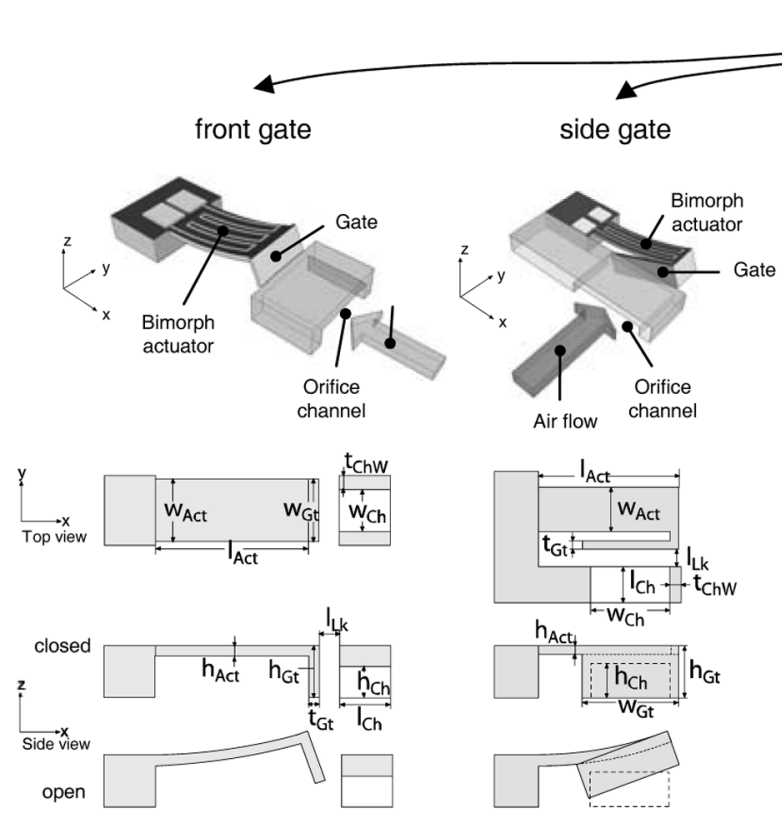

(A)

(B)
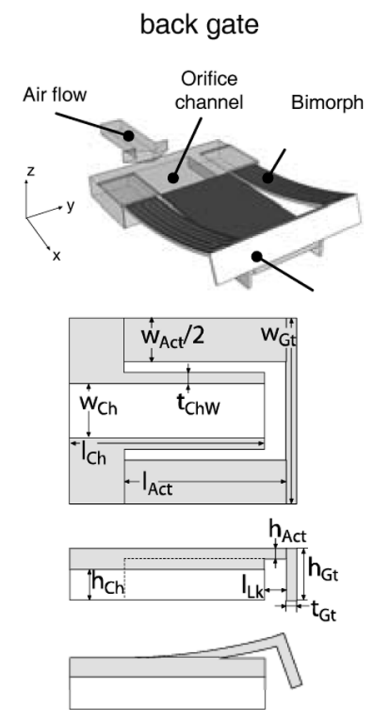

(C)

Fig. 1. ( $\alpha$ and $\beta$ ): Two orifice/obstruction configurations common in microsystems. (a)-(c): Three micromachined cross-flow valve variations. (a) Seat valve: the obstruction is called the boss. (b) Cross-flow or gate valve: the obstruction is called the gate. (A-C): Three possible out-of-plane gate microvalves. (A) Front-gate valve. (B) Side-gate valve. (C) Back-gate valve. The orifice channel is drawn transparently for clarity.

TABLE I

Classification of Micromachined Gate Valves

\begin{tabular}{lllll}
\hline Figure & gate orientation & gate movement & flow direction & fluid \\
\hline 1a & in plane & in plane & out of plane & gas [18], [19] \\
1b & out of plane & in plane & in plane & liquid [20], [21] \\
1c & out of plane & out of plane & in plane & gas (this work) [22] \\
\hline
\end{tabular}

for electrical connections, which must make their way out of the package.

\section{Gate-Microvalve Design}

Table I gives an overview of published micromachined gate valves. It also classifies the gate valves by the orientation of the gate and the plane in which it moves. Cross-flow valve actuation in the world of planar microsystem technology has three basic configuration schemes in terms of flow and actuation direction. The first configuration [Fig. 1(a)] features in-plane actuation and out-of-plane flow. This configuration is used in the valve design by Walters et al. and Williams et al. [18], [19]. The second configuration [Fig. 1(b)] features in-plane actuation and in-plane 
TABLE II

COMPARISON OF THE KEY FEATURES FOR THE DIFFERENT VALVE DESIGNS IN FOCUS

\begin{tabular}{|c|c|c|c|c|c|}
\hline & \multicolumn{2}{|c|}{ design requirements for optimum... } & \multicolumn{3}{|c|}{ designs in focus } \\
\hline & flow performance & foot print & side gate & front gate & back gate \\
\hline leak gap $\left(l_{L k}\right)$ & minimize & negligible & small & large & small \\
\hline inner orifice channel footprint $\left(w_{C h} \times l_{C h}\right)$ & negligible & minimize & small & small & large \\
\hline mechanical stability & maximize & minimize & medium & good & good \\
\hline footprint area & maximize & minimize & small & medium & large \\
\hline actuator length $\left(l_{A c t}\right)(\propto$ stroke $)$ & maximize & minimize & long & medium & medium \\
\hline
\end{tabular}

flow [22]. This approach has been used for on-chip liquid control [20], [21] but has - to the authors' knowledge—not yet been reported for gas control. The third configuration [Fig. 1(c)] features out-of-plane actuation and in-plane flow. This configuration is used in the novel knife-gate microvalve introduced in this paper.

The main reason for using a cross-flow valve is that the actuator does not need to counteract the static pneumatic force. This eases the requirements since one does not have to overcome the force exerted by the static pressure. The main advantage of using out-of-plane gate orientation is the low lateral footprint area. No chip area has to be reserved for the movement of the actuator. The support substrate material (see Fig. 3) onto which the device needs to be attached is not structured, making its cost negligible. As stated previously, friction and stiction between sliding microstructures must be avoided. Therefore, there has to be a gap between the gate and the orifice which results in a closed-state leakage. Process parameters and the direction of the gate movement can affect the size of the leak gap, so the choice of the valve design depends on the leak tolerance of the intended application.

Fig. 1(A)-(C) shows the three possible gate-actuator configurations for microvalves with out-of-plane gate movement: the front, side, and back gate, respectively. In the following discussion, $w_{q}, l_{q}, h_{q}$, and $t_{q}$ will be used to indicate the width, length, height, and thickness of feature $q$, respectively. The following key features will be taken into account.

$\begin{array}{ll}\text { Ch } & \text { Inner dimensions of the orifice nozzle/channel } \\ \text { that is cut off by the gate. }\end{array}$

Having defined these quantities, we turn to the effect of these parameters. Table II shows the general requirements on these key features to obtain an optimum flow performance or a minimum footprint area, respectively. The table also informs about the typical dimension of each feature in each of the three designs in focus.

Friction between sliding microstructures must be avoided. Therefore, there has to be a gap with size $l_{\mathrm{Lk}}$ between the gate and the orifice which results in a closed-state leakage. This leak gap should be minimized, reducing the leak flow in the closed state, improving the flow performance of the valves and minimizing the valve's footprint area. Microfabrication allows to minimize the gap. For the side-gate and back-gate valve, this leakage gap is only determined by the manufacturing process (etch aspect ratio), in this case the deep reactive ion etching (DRIE)-tool used, resulting in $l_{\mathrm{Lk}} \approx 10 \mu \mathrm{m}$. For the front-gate valve, the gap is determined by the operation, not by the fabrication. The latter design requires the largest orifice-gate spacing, due to the outward rotation of the gate during upward movement, which results in a higher closed-state leakage. For applications that tolerate little leakage, therefore, the back and side-gate variants are preferred.

The inner orifice channel's footprint area $\left(w_{\mathrm{Ch}} \times l_{\mathrm{Ch}}\right)$ has a minor effect on the flow performance, but it is important in terms of the valve's footprint area. The channel footprint should be as small as possible to minimize the overall footprint area. The back-gate valve design has the largest inner orifice channels footprint area of the three designs but compensates by combining two major advantages: a leakage gap determined by fabrication rather than operation and the best mechanical stability.

The mechanical stability of the bimorph actuator directly influences the flow performance of the valves. However, strengthening typically results in increased footprint area of the actuator beams. The side-gate valve is the mechanically weakest design, since the pressure force exerted on the closed gate results in a large torsion along the actuator. However, the side gate has the smallest footprint area, making it suitable for applications with small pressure ranges.

Another limiting factor for the flow performance is the footprint area itself. A larger footprint area typically allows a larger flow nozzle, thus an increased flow performance. However, an increased footprint area also reduces the cost-efficiency of the valve.

The actuator length $\left(l_{\mathrm{Act}}\right)$ is proportional to the stroke of the actuator and the maximum orifice height possible. A large stroke results in a higher cross-sectional area of the orifice channel $\left(w_{\mathrm{Ch}} \times h_{\mathrm{Ch}}\right)$ and an improved flow performance. However, a longer actuator also results in mechanically weaker actuators, sensitive to the pressure force exerted on the gate. The side gate has the longest actuator, making it the mechanically weakest of the three valves.

\section{Performance of a Pressure Controller WITH LEAKY VALVES}

In this section, we explain the performance of a leaky pressure controller as a motivation for the development of a leaky valve. We show that even with significant leaks of up to $20 \%$, the pressure controller can still provide $94 \%$ of its pressure range. The principle of the pressure controller, for which the microvalve in this work is designed, is illustrated in Fig. 2(a). It consists of two valves connected in series. By controlling the two valves, the work pressure $P_{\text {work }}$ can be regulated between the supply 


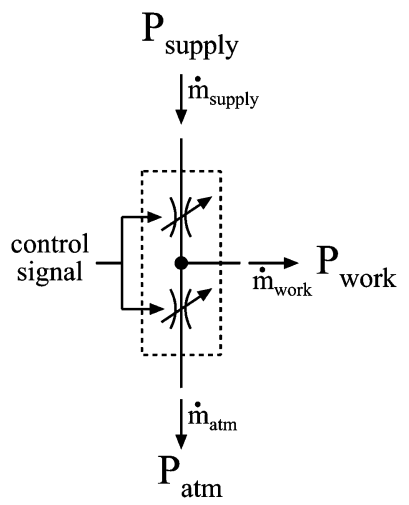

(a)

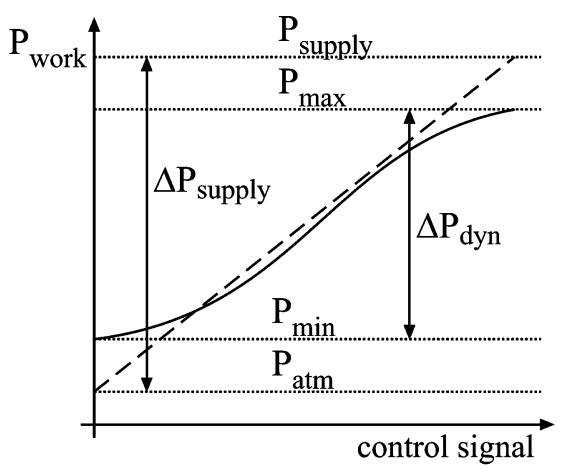

(b)

Fig. 2. Functional representation of a pressure-control element (left) and its pressure characteristics (right). The dashed line in the plot indicates the ideal behavior, while the solid line indicates a typical actual performance.

pressure $P_{\text {supply }}$ and the atmospheric pressure $P_{\text {atm }}$. The effect of valve leak on the pressure controller [illustrated in Fig. 2(b)] influences the controller's static pneumatic energy loss and reduces the dynamic pressure range $\Delta P_{\mathrm{dyn}}$ of the device to a maximum possible pressure $P_{\max }$ and a minimum possible pressure $P_{\min }$

$$
\Delta P_{\text {dyn }}=P_{\max }-P_{\min }<\Delta P_{\text {supply }}=P_{\text {supply }}-P_{\text {atm }} .
$$

The influence of the valve leak on the performance of the full pressure converter can be quantified as follows. In the leak gap, the typical flow conditions result in a Reynolds number $R_{e} \geq 200$, and a boundary layer thickness $\delta=\sqrt{(\mu L / \rho \bar{u})}<$ $\left(l_{\mathrm{Lk}} / 2\right)$, where $\mu$ and $\rho$ are the viscosity and the density of air, respectively, $\bar{u}$ is the average flow velocity, and $L=\left(h_{\mathrm{Gt}}-\right.$ $\left.h_{\mathrm{Ch}}\right), t_{\mathrm{ChW}}$ is the path length for the leak flow. Therefore, for moderate pressure drop, we can approximate both the main flow and the leak flow as subsonic isentropic flow through a sudden expansion [23].

The mass flow

$$
\dot{m} \propto A_{c s} P_{\text {supply }}\left(\frac{P_{\text {atm }}}{P_{\text {supply }}}\right)^{1 / \gamma} \sqrt{1-\left(\frac{P_{\text {atm }}}{P_{\text {supply }}}\right)^{(\gamma-1) / \gamma}}
$$

with $A_{c s}$ being the minimal cross-sectional area of the flow path and $\gamma$ the gas specific heat ratio. The normalized leak ratio can then be defined as

$$
\begin{aligned}
\eta & =\frac{\dot{m}_{\mathrm{closed}}}{\dot{m}_{\mathrm{open}}}=\frac{A_{c s, \text { closed }}}{A_{c s, \text { open }}}=\frac{\left(2 h_{\mathrm{Ch}}+w_{\mathrm{Ch}}\right) \cdot l_{\mathrm{Lk}}}{h_{\mathrm{Ch}} \cdot w_{\mathrm{Ch}}} \\
& \approx \frac{l_{\mathrm{Lk}}}{h_{\mathrm{Ch}}} \text { for } w_{\mathrm{Ch}} \gg h_{\mathrm{Ch}} .
\end{aligned}
$$

The indices closed and open refer to the conditions and dimensions at the gate-nozzle spacing in the closed state and the maximum nozzle opening in the open state, respectively.

For a pressure controller, as illustrated in Fig. 2, containing two identical control valves with leak rate $\eta$, one can calculate $P_{\min }$ and $P_{\max }$ by using the mass flow continuity equation

$$
\dot{m}_{\text {supply }}=\dot{m}_{\text {work }}+\dot{m}_{\mathrm{atm}}
$$

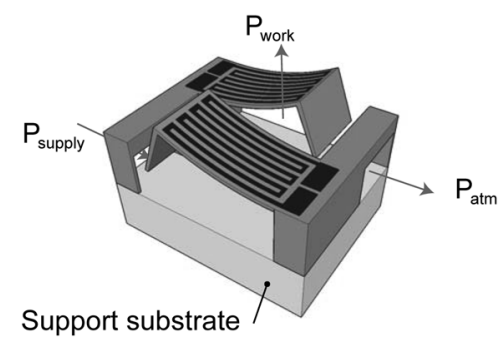

Fig. 3. Process flow for prototype fabrication.

At zero work flow and $A_{c s, \text { supply }}=\eta \cdot A_{c s, \text { vent }}, P_{\text {work }}=$ $P_{\min }$ when the vent port is open and the supply port is closed. If the vent port is closed and the supply port is open, $P_{\text {work }}=$ $P_{\max }$ and $A_{c s, \text { vent }}=\eta \cdot A_{c s, \text { supply }} \cdot P_{\min }$ and $P_{\max }$ are thus the respective solutions of

$$
\begin{aligned}
\eta \cdot P_{\text {supply }} & \left(\frac{P_{\text {min }}}{P_{\text {supply }}}\right)^{1 / \gamma} \sqrt{1-\left(\frac{P_{\text {min }}}{P_{\text {supply }}}\right)^{(\gamma-1) / \gamma}} \\
& =P_{\min }\left(\frac{P_{\text {atm }}}{P_{\min }}\right)^{1 / \gamma} \sqrt{1-\left(\frac{P_{\text {atm }}}{P_{\min }}\right)^{(\gamma-1) / \gamma}}
\end{aligned}
$$

and

$$
\begin{aligned}
P_{\text {supply }} & \left(\frac{P_{\max }}{P_{\text {supply }}}\right)^{1 / \gamma} \sqrt{1-\left(\frac{P_{\max }}{P_{\text {supply }}}\right)^{(\gamma-1) / \gamma}} \\
& =\eta \cdot P_{\max }\left(\frac{P_{\text {atm }}}{P_{\max }}\right)^{1 / \gamma} \sqrt{1-\left(\frac{P_{\text {atm }}}{P_{\max }}\right)^{(\gamma-1) / \gamma}} .
\end{aligned}
$$

Numerically solving these equations for $100 \mathrm{kPa}$ (relative), supply pressure shows that, for a leak rate $\eta=20 \%, P_{\max }=$ $98.15 \mathrm{kPa}$ and $P_{\min }=3.76 \mathrm{kPa}$, which gives a pressure range $\left(\left(\Delta P_{\text {dyn }}\right) /\left(\Delta P_{\text {supply }}\right)\right) \approx 94 \%$. Fig. 3 illustrates how two front-gate valves can be combined to form a pressure controller.

\section{FABRICATION}

Prototypes were fabricated to evaluate the feasibility of silicon-gate microvalves with out-of-plane actuation. The dimensions of the devices were varied and some typical dimensions are stated in Table III. Deep reactive ion etching was used to 
TABLE III

TYPICAL DIMENSIONS OF THE MICROVALVES

\begin{tabular}{llc} 
Feature & Parameter & Typical dimension in $\mu \mathrm{m}$ \\
\hline Orifice channel & width $w_{C h}$ & 1000 \\
& height $h_{C h}$ & 250 \\
length $l_{C h}$ & $1000-3000$ \\
Orifice channel wall & thickness $t_{C h}$ & 1000 \\
\hline \multirow{2}{*}{ Gate } & width $w_{G t}$ & $1000-2200$ \\
& height $h_{G t}$ & 400 \\
& thickness $t_{G t}$ & 70 \\
\hline \multirow{2}{*}{ Leak gap } & length $l_{L k}$ & 10 \\
\hline \multirow{2}{*}{ Actuator } & width $w_{A c t}$ & $500-1000$ \\
& height $h_{A c t}$ & 11.5 \\
& length $l_{A c t}$ & 2000 \\
\hline
\end{tabular}

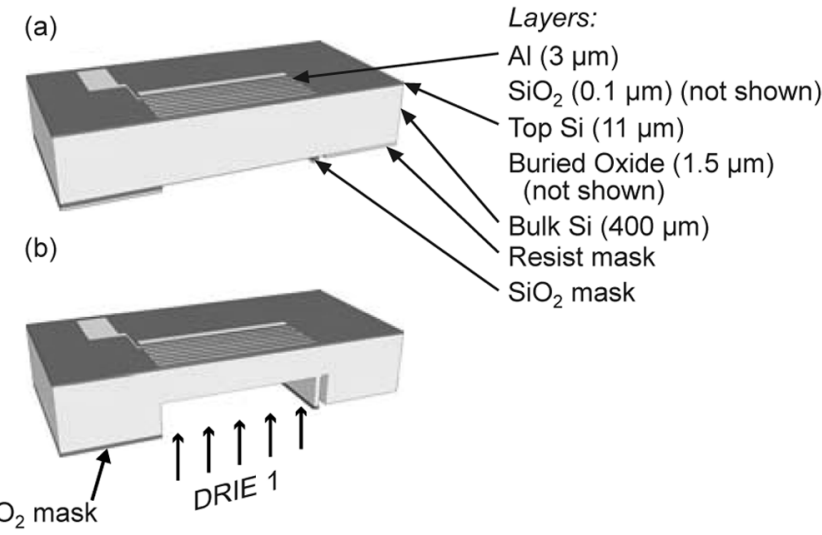

(c)

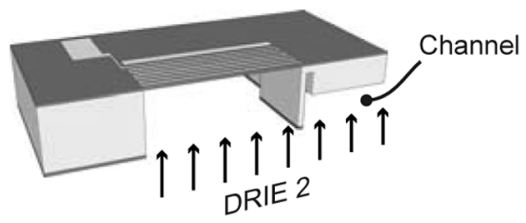

(d)

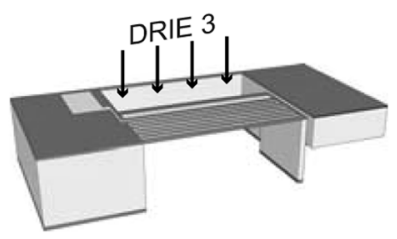

Fig. 4. Scanning electron microscope (SEM) picture of the three types of fabricated knife-gate microvalves.

define the structure, while the intrinsic tensile stress of the sputtered aluminum layer provided the initial deflection. The aluminum-silicon bimorph was designed to be actuated by direct resistive heating of the aluminum.

Fig. 4 shows the process flow for a front-gate device. The starting point is a silicon-on-insulator (SOI) wafer with a $11.5-\mu$ $\mathrm{m}$ device layer and $1-\mu \mathrm{m}$ buried oxide layer on top of $400-\mu$ $\mathrm{m}$-thick bulk silicon. In the first step, the wafer is wet oxidized to create a $1-\mu \mathrm{m}$-thick back-side masking layer. Then, the oxide on the front side is etched away and regrown to obtain a 100-nm layer to electrically insulate the silicon from the subsequently sputtered $3-\mu \mathrm{m}$-thick aluminum layer.

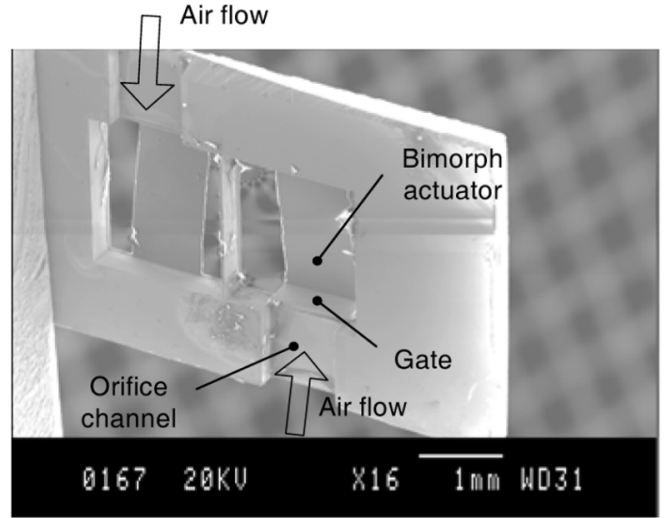

(a)

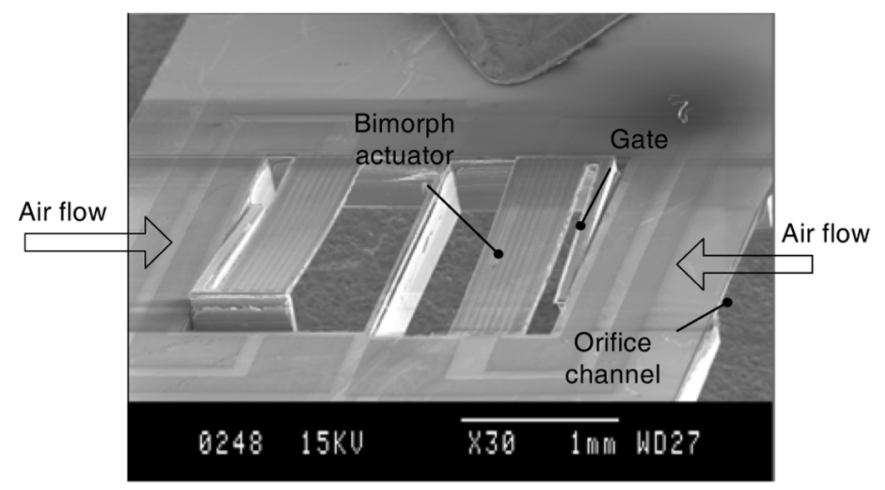

(b)

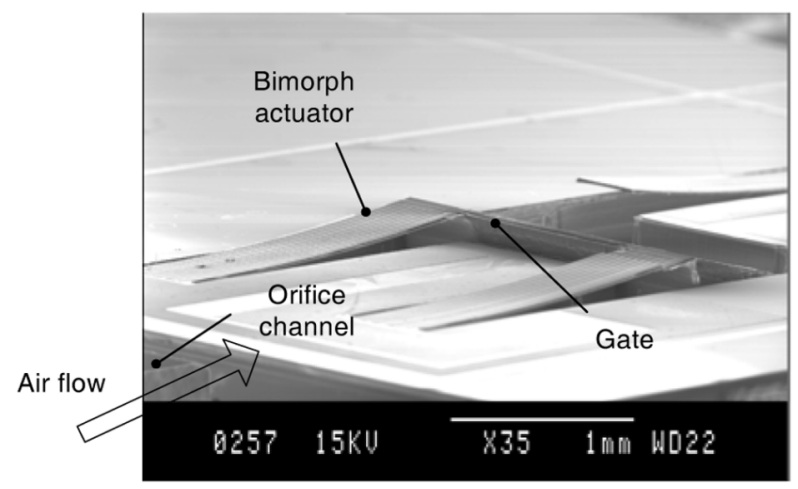

(c)

Fig. 5. Conceptual drawing of the combination of two cross-flow valves to form an $\mathrm{I} / \mathrm{P}$ converter or three-way valve.
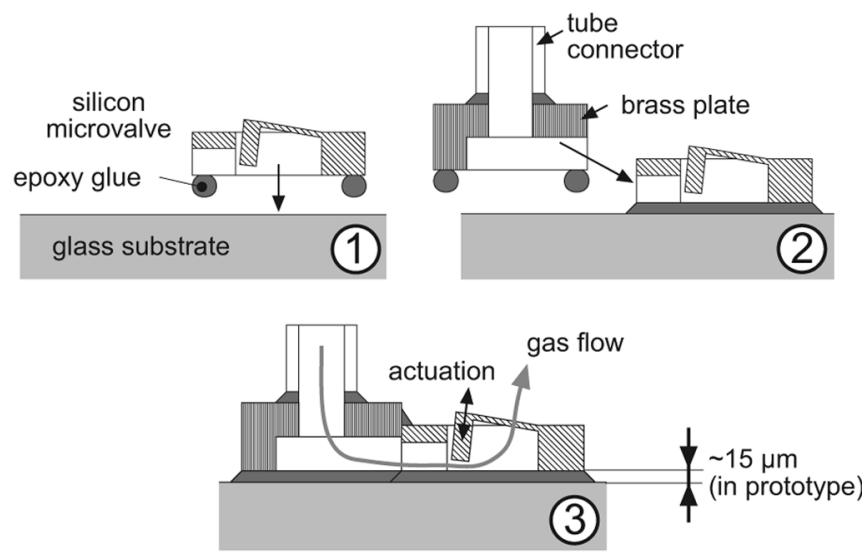

Fig. 6. (Not to scale) Cross-sectional view of the prototype assembly of the pneumatic interconnection at the side of the chip used for testing. 


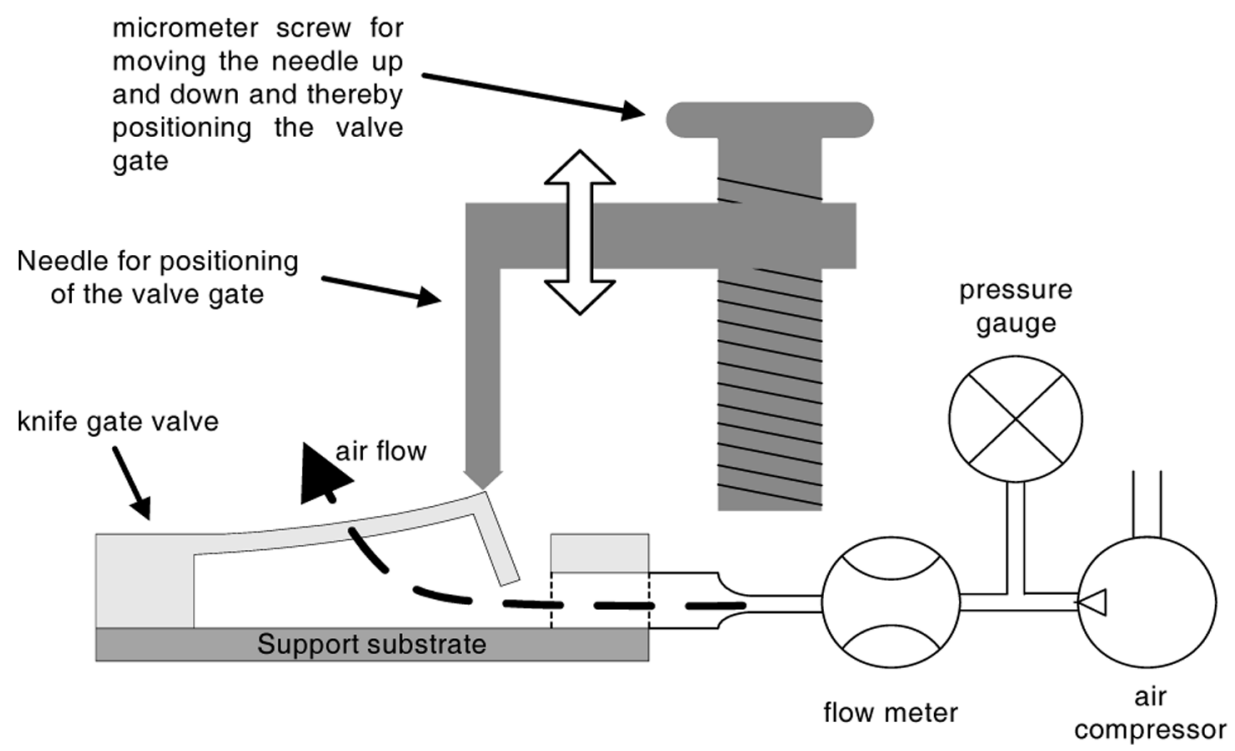

Fig. 7. (Not to scale) Measurement setup with an external manipulator consisting of a micrometer screw with a needle moving up and down and positioning the gate of the valve.

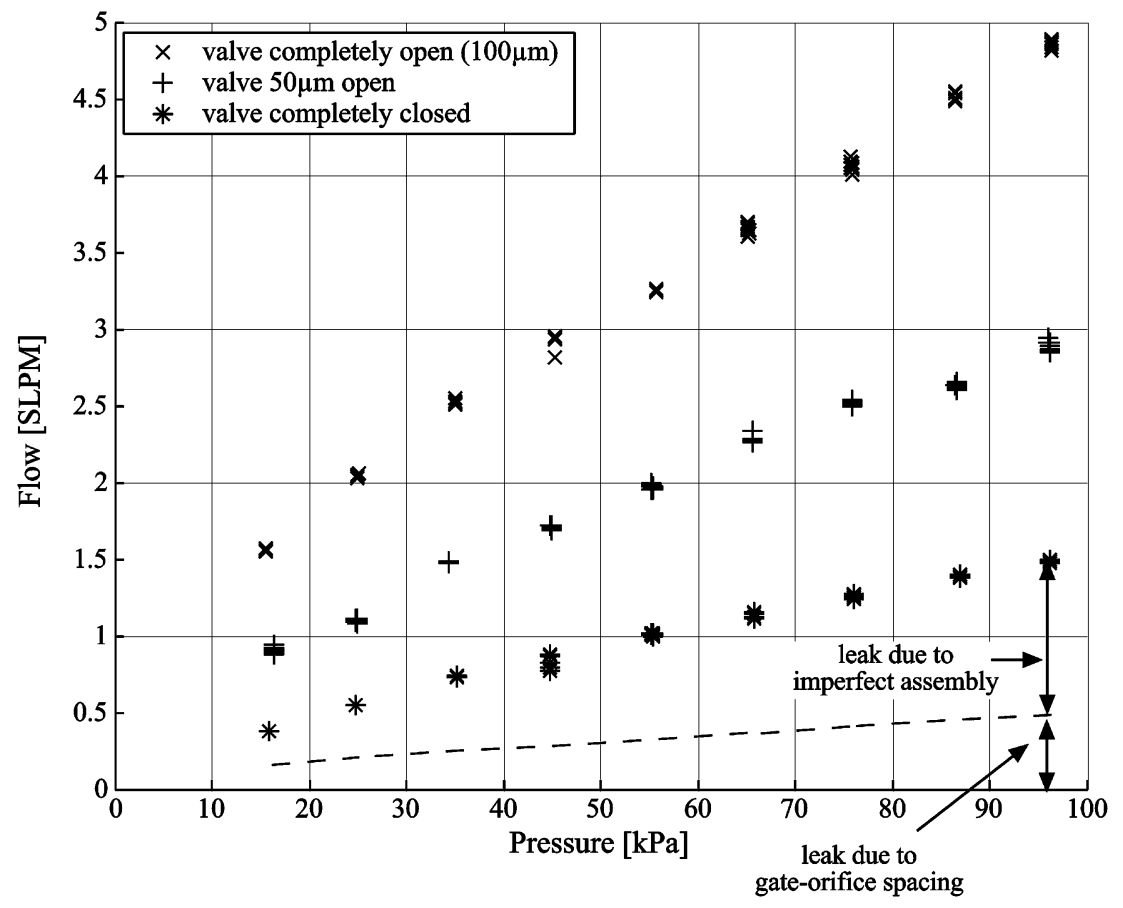

Fig. 8. Measured pressure-flow characteristics. The dashed line indicates the expected leak caused by the spacing between orifice and gate.

The aluminum is patterned and wet-etched to form the heaters. After this, the back side is patterned in two steps to define the orifice channels and bimorph beams. The first mask defines the bimorph beams and the orifice channels and is etched in the oxide. The second mask covers the orifice channels with thick resist. This is the situation illustrated in Fig. 4(a). The back side is then etched using DRIE in two steps, where the thick resist for the second mask is removed halfway, causing the orifice channels to be etched only during the second step. This is shown in Fig. 4(b) and (c). Finally, a front-side DRIE frees the devices and a buffered hydrofluoric (BHF) etch, using the same photoresist mask, removes all unnecessary oxide. In some cases, reactive ion etching (RIE) lag hindered the complete etching of the narrow gap between the gate and the orifice channel. In those cases, an additional DRIE from the front was performed to "cut" free the gates. After this, the devices were diced. The final device is shown in Fig. 4(d). Fig. 5 shows the fabricated gate microvalves.

\section{EXPERIMENTS}

For our prototype evaluation, an interfacing method was developed, shown in Fig. 6. A brass adapter between the chip and the tube was used, thereby trimming the use of silicon chip area to its essence, namely the valve actuation. The assembly method was evaluated on a valve with the gate removed by ramping up the pressure until the assembly ruptured. This occurred at 6 bar 
and happened at the glue/glass interface. The authors believe that the limited pressure tolerance (6 bar) of the test package can be significantly improved with a reasonable effort in glue selection and substrate preparation.

For pressure-flow characterization, the valve outlet was open to atmosphere. The inlet was connected to a pressure source and the pressure and flow speed were measured. The bimorph beam was found not to be strong enough to withstand the vibration and torsional forces caused by the flow. Therefore, to evaluate the pressure-flow characteristics, an external manipulator was used to hold the gate in the desired position. The exact gate position could be determined via the micrometer screw of the external manipulator, as illustrated in Fig. 7.

The measured pressure-flow characteristics, shown in Fig. 8, confirm the valve's potential for controlling large flows: A flow orifice size of $1000 \times 200 \mu \mathrm{m}^{2}$ allows a flow change of $\Delta Q=$ 3.4 SLPM at a pressure change of $\Delta P=95 \mathrm{kPa}\left(P_{\text {in }}=195 \mathrm{kPa}\right.$, $\left.P_{\text {out }}=100 \mathrm{kPa}\right)$ on an active chip area of only $2.3 \times 3.7 \mathrm{~mm}^{2}$ for a single valve. Fig. 8 also shows the two components of the relatively large leak flow, which results mainly from the additional leakage gap of $15 \mu \mathrm{m}$ caused by the imperfect assembly (Fig. 6).

The origin of leak flow (the flow when the device is completely closed) can be attributed to two causes: About one third of it is caused by the gate-orifice spacing and is inherent to the tolerances of the fabrication process, while the remaining two thirds are due to the manual assembly and can be resolved by better bonding procedures. The leak flow is considered acceptable for the intended applications. To the authors' knowledge, the flow performance per footprint area is at least an order of magnitude higher than that of nongate microvalves designed for similar flows [16].

\section{CONCLUSION}

We have presented a novel microvalve concept for pressure-control applications. The design is the key element in a truly miniaturized micromachined high-performance pneumatic control element. The pressure-flow performance per chip area of the demonstrator valve presented is dramatically increased using out-of-plane actuators with in-plane orifice channels. Three different actuator-gate configurations were introduced and discussed. A demonstrator structure based on an aluminum-silicon bimorph was fabricated. The device was tested and the flow-pressure and flow-gate opening performance were measured. The valve flow can be controlled gradually through the gate position. The authors believe that silicon-aluminum bimorph beams produced in the way described will not be robust enough for controlling large pressures and flows. Alternative integrated actuation mechanisms are under consideration.

\section{REFERENCES}

[1] P. J. Hesketh, J. S. Bintoro, and L. Luharuka, , J. K. H. Baltes and G. K. Fedder, Eds., "Microvalve for fuel cells and miniature gas chromatographic system," in Sensors Update 13. Weinheim, Germany: Wiley, 2004, pp. 233-302.

[2] L. Yobas, M. A. Huff, F. J. Lisy, and D. M. Durand, "A novel bulk micromachined electrostatic microvalve with a curved-compliant structure applicable for a pneumatic tactile display," J. Microelectromech. Syst., vol. 10, no. 2, pp. 187-196, Jun. 2001.

[3] M. Huff, J. Gilbert, and M. Schmidt, "Flow characteristics of a pressure balanced microvalve," in Proc. 7th Int. Conf. Solid State Actuators (Transducers), Yokohama, Japan, 1993, pp. 98-101.
[4] E. T. Carlen and C. H. Mastrangelo, "Parrafin actuated surface micromachined valve," in Proc. IEEE Int. Conf. Microelectromech. Syst. (MEMS), Tech. Dig., Jan. 2000, pp. 381-385.

[5] —_, "Surface micromachined parrafin-actuated microvalve," J. Microelectromech. Syst., vol. 11, no. 5, pp. 408-420, Oct. 2002.

[6] Rich and Wise, "A high-flow thermopneumatic actuated microvalve with improved efficiency and integrated state sensing," J. Microelectromech. Syst., vol. 12, no. 2, pp. 201-208, Apr. 2003.

[7] S. Messner, M. Muller, J. Schaible, H. Sandmaier, and R. Zengerle, "3-way microvalve for pneumatic applications fabricated by silicon micromachining," in Proc. Microelectromech. Syst. (MEMS)—1998/ ASME Int. Mech. Eng. Congr. Exposition, Anaheim, CA, 1998, pp. 159-164.

[8] M. Shikida, K. Sato, S. Tanaka, Y. Kawamura, and Y. Fujisaki, "Electrostatically driven gas valve with high conductance," J. Microelectromech. Syst., vol. 3, no. 2, pp. 76-80, Jun. 1994.

[9] K. Sato and M. Shikida, "An electrostatically actuated gas valve with an S-shaped film element," J. Micromech. Microeng., vol. 4, no. 4, pp. 205-209.

[10] F. Pourahmadi, L. Christel, K. Petersen, J. Mallon, and J. Bryzek, "Variable-flow microvalve structure fabricated with silicon fusion bonding," in Proc. IEEE Solid-State Sens. Actuator Workshop, Tech. Dig., Hilton Head Island, SC, 1990, pp. 78-81.

[11] A. D. Johnson, "Vacuum-deposited TiNi shape memory film: Characterization and applications in microdevices," J. Micromech. Microeng. vol. 1, no. 1, pp. 34-41.

[12] A. K. Henning, J. Fitch, D. Hopkins, L. Lilly, R. Faeth, E. Falsken, and M. Zdeblick, "A thermopneumatically actuated microvalve for liquid expansion and proportional control," in Int. Conf. Solid-State Sens. Actuators, Tech. Dig., Chicago, IL, 1997, vol. 2, pp. 825-828.

[13] D. C. Roberts, L. Hanqing, J. L. Steyn, O. Yaglioglu, S. M. Spearing, M. A. Schmidt, and N. W. Hagood, "A piezoelectric microvalve for compact high-frequency, high-differential pressure hydraulic micropumping systems," J. Microelectromech. Syst., vol. 12, no. 1, pp. 81-92, Feb. 2003.

[14] S. Kluge, G. Neumayer, U. Schaber, M. Wackerle, M. Maichl, P. Post, M. Weinmann, and R. Wanner, "Pneumatic silicon microvalves with piezoelectric actuation," in Proc. 11th Int. Conf. Solid-State Sens. Actuators, Tech. Dig., Munich, Germany, 2001, vol. 2, pp. 924-927.

[15] W. van der Wijngaart, H. Ask, P. Enoksson, and G. Stemme, "A high-stroke, high-pressure electrostatic actuator for valve applications," Sens. Actuators A, Phys., vol. A100, no. 2-3, pp. 264-271.

[16] W. van der Wijngaart, A. Thorsén, and G. Stemme, "A seat microvalve nozzle for optimal gas flow capacity at large controlled pressure," $J$. Microelectromech. Syst., vol. 14, no. 2, pp. 200-206, Apr. 2005.

[17] R. Silvestrini, "Solenoid valve with stroke insensitive port," U.S. Patent 3729025, 1973.

[18] M. Walters, V. Dhuler, R. Mahadevan, A. Cowen, R. Wood, E. Hill, and I. Kao, "A silicon micromachined gate valve," presented at the 1998 Solid-State Sens. Actuators Symp., SC, 1998.

[19] K. R. Williams, N. I. Maluf, E. N. Fuller, R. J. Barron, D. P. Jaeggi, and B. P. van Drieënhuizen, "A silicon microvalve for the proportional control of fluids," in Proc. Transducers '99, Sendai, Japan, 1999, pp. 1804-1807

[20] A. P. Papavasiliou, D. Liepmann, and A. P. Pisano, "Fabrication of a free floating silicon gate valve," in Proc. Int. Mech. Eng. Congr. Exposition (IMECE), Nashville, TN, 1999, pp. 435-440.

[21] J. A. Frank and A. P. Pisano, "Low-leakage micro gate valves," in Proc. 12th Int. Conf. Solid-State Sens. Actuators Microsyst. (Transducers'03), Tech. Dig., Boston, MA, 2003, vol. 1, pp. 143-146.

[22] W. van der Wijngaart, A. S. Ridgeway, and G. Stemme, "A micromachined knife gate valve for high-flow pressure regulation applications," in Proc. 12th Int. Conf. Solid-State Sens. Actuators Microsyst. ( Transducers'03 ), Tech. Dig., Boston, MA, 2003, vol. 2, pp. 1931-1934.

[23] F. M. White, Fluid Mechanics, 4th ed. New York: McGraw-Hill, 1999, pp. 586-587.

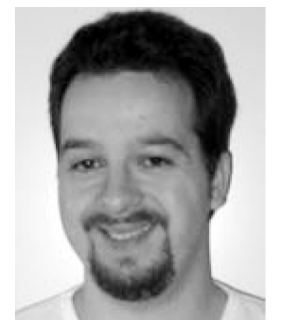

Sjoerd Haasl (S'03-M'04) received the M.Sc degree with high honors in electrotechnical engineering, specializing in automation and computer systems, from the University of Leuven, Leuven, Belgium, in 1999 and the Ph.D. degree in microsystem technology from the Royal Institute of Technology, Stockholm, Sweden, in 2005.

Currently, he is a Research Engineer at IMEGO $\mathrm{AB}$, Gothenburg, Sweden. His current research interests lie in the fields of inertial sensors, flow sensors, and chemical sensors. 


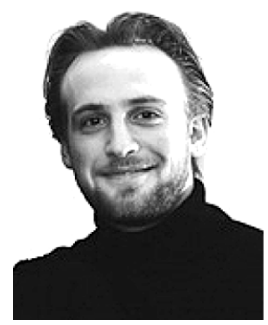

Stefan Braun was born in Germany in 1980 . He received the Dipl.-Ing. (FH) degree in microsystem technology from the University of Applied Sciences, Zweibrücken, Germany, in 2003 and currently is working towards the Ph.D. degree in microelectromechanical systems (MEMS) at the School of Electrical Engineering, Royal Institute of Technology, Stockholm, Sweden.

His research focus is on microswitch arrays and microvalves.

Anthony S. Ridgeway was awarded a Fulbright Grant for graduate study and research for working at the Royal Institute of Technology in Stockholm, Sweden, during the academic year 2002-2003 and received the M.Sc. degree in mechanical engineering form the Iowa State University, Ames.

Samir Sadoon was born in 1979. He received the M.Sc. degree in electrical engineering from the Royal Institute of Technology, Stockholm, Sweden, in 2004.

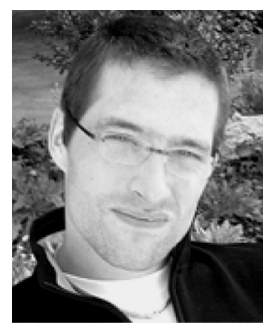

Wouter van der Wijngaart (M'06) received the M.Sc. degree in electrotechnical engineering, the degree of philosophic academy, and the mathematics education degree, all from the Katholieke Universiteit Leuven, Leuven, Belgium, in 1996 and the $\mathrm{Ph} . \mathrm{D}$. degree in microsystem technology from the Royal Institute of Technology (KTH), Stockholm, Sweden, in 2002.

In 2005, he became an Associate Professor at KTH. He is currently leading the micro- and nanofluidics research at the Microsystem Tech- nology Laboratory at KTH. He is the overall Project Manager of the European Framework Six (FP6) project "Q2M-batch integration of high quality materials to microsystems" and leading the microfluidics work of the European FP6 project "SABIO-ulitra high sensitive slot waveguide biosensor." He is also a cofounder of three companies in the fields of mobile parking payments, microvalving, and microfuel cells.

Prof. van der Wijngaart received, together with Fredric Ankarcrona, the Cap Gemini Innovation Award in the 1999 European Business Plan of the Year Competition. He was also awarded the Swedish Innovation Cup 2001, together with Prof. Andersson.

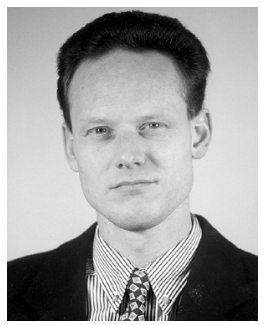

Göran Stemme (M'98-SM'04-F'06) received the M.Sc. degree in electrical engineering in 1981 and the Ph.D. degree in solid state electronics in 1987, both from the Chalmers University of Technology, Gothenburg, Sweden.

In 1981, he joined the Department of Solid State Electronics, Chalmers University of Technology where in 1990, he became an Associate Professor (Docent) heading the silicon sensor research group. In 1991, he was appointed a Professor at The Royal Institute of Technology, Stockholm, Sweden, where he heads the Microsystem Technology group at the Department of Signals, Sensors and Systems. He has published more than 100 research journal and conference papers and has been awarded eight patents. His research is devoted to microsystemtechnology based on micromachining of silicon.

Dr. Stemme was a member of the International Steering Committee of the Conference series IEEE Microelectromechanical Systems (MEMS), between 1995 and 2001, and he was a General Co-chair of that conference in 1998. $\mathrm{He}$ is a member of the Editorial Board of the IEEE/ASME JouRNAL OF MicROELECTROMECHNICAL SYSTEMS and of the Royal Society of Chemistry journal Lab On A Chip. In 2001, he won, together with two colleagues, the final of the Swedish Innovation Cup. 